\title{
Sommerdermatosen durch UV-Strahlung
}

\section{Summer Dermatoses Caused by UV-Irradiation}

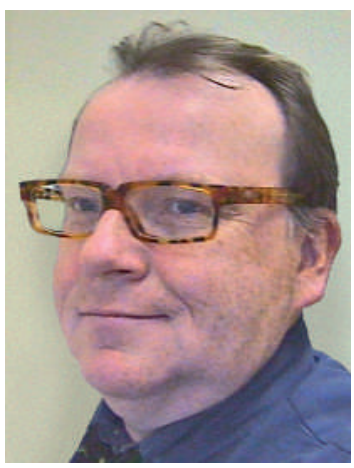

Prof. Dr. med. habil. L. Kowalzick
Der Sommer neigt sich dem Ende zu, die Tage werden wieder deutlich kürzer. In den letzten Monaten sahen wir vermehrt Patienten mit Lichtdermatosen bzw. lichtprovozierten oder -aggravierten Dermatosen.

Einen ersten Gipfel hatte die polymorphe Lichtdermatose, die mit Abstand häufigste Erkrankung dieser Gruppe (über 60\% aller Patienten, die wegen „Lichtproblemen“ - abgesehen von Sonnenbränden - vorstellig werden [Kowalzick L et al. Akt Dermatol 1994; 20: 173-177]), bereits im späteren Frühjahr, einige Stunden nach der ersten intensiveren Besonnung des Jahres. Aber etwa bei $1 / 3$ der Patienten traten die Hautveränderungen dieser Erkrankung erst im Hochsommer bzw. im Fernurlaub unter südlicher Sonne auf. Die Prävalenz beträgt in unseren Breiten ca. $15 \%$; Frauen sind ca. dreimal so häufig betroffen wie Männer, mehr als die Hälfte der Patienten gehört dem Hauttyp I oder II nach Fitzpatrick an, und die meisten Patienten erkranken erstmals im 3. Lebensjahrzehnt. Die Erkrankung, die beim individuellen Patienten stets monomorph ist, unterscheidet sich morphologisch zwischen verschiedenen Patienten klinisch zum Teil deutlich (papulöser, plaqueartiger, papulovesikulöser Typ mit Untertypen). Prädilektionsstellen sind Dekolletée, Unterarme, also Hautpartien, die - anders als das Gesicht - nicht ständig dem Licht ausgesetzt sind. Typisch ist ein Gewöhnungseffekt an das Licht (Hardening) binnen kurzer Zeit.

Phototoxische oder photoallergische Dermatitiden (ca. 10\% der vorstelligen Patienten) können exogen z.B. nach Hautkontakt mit pflanzlichen Photosensibilisatoren, mit Duftstoffen, mit Externa (z.B. auch Diclofenac [Solaraze ${ }^{\circledR}$ ], Kowalzick L, Ziegler H. Akt Dermatol 2006; 32: 50-52) oder häufiger paradoxerweise mit Inhaltsstoffen von topischen Lichtschutzmitteln ausgelöst werden. Endogene Auslöser sind vor allem Medikamente (Antibiotika, Psychopharmaka, Antiphlogistika, Phytopharmaka), selten Nahrungsergänzungsmittel und Nutritiva.

Bei Patienten mit Lupus erythematodes (ca. 4\% der vorstelligen Patienten) können die lichtprovozierten Hautveränderungen oft erst mehrere Wochen oder wenige Monate nach intensiverer Besonnung klinisch manifest werden, so dass der Zusammenhang mit der Belichtung gelegentlich für den Patienten und den Arzt verborgen bleibt.
Es wird jetzt Zeit, die Diagnostik von Lichtdermatosen (Kowalzick L, Mensing H, Wagner G (Hrsg). Praxis der Lichtdermatosen. Diagnostik, Therapie und Prävention. Uni.-Med., 2000 Bremen: 21 -32) in der lichtarmen Jahrszeit (am besten von Dezember bis Februar) auf der lichtentwöhnten Haut zu planen. Zunächst sollte eine Bestimmung der minimalen erythematogenen Dosis (MED), jeweils getrennt für UV-A und UV-B, erfolgen. Bei pathologischen Erythemschwellen wäre in erster Linie an eine chronisch- aktinische oder eine phototoxische Reaktion zu denken. Die Industrie bietet Geräte zur vereinfachten, personaleinsatz-schonenden MED-Diagnostik an. Zum Ausschluss einer Lichturtikaria sollte außer der 24-Stundenablesung auch eine Sofortablesung nach 20 bis 40 Minuten erfolgen.

Bei unauffälligen Erythemschwellen sollte danach eine Provokationstestung an zumindest drei aufeinanderfolgenden Tagen, gleichfalls getrennt für UV-A und UV-B, erfolgen.

Isomorphe Herde lassen sich bei der polymorphen Lichtdermatose in über $80 \%$ der Fälle provozieren. Auslösend ist hierbei in ca. $85 \%$ UV-A, in ca. $10 \%$ UV-B und UV-A und in ca. 5\% UV-B allein. Die Histologie ist typisch, aber meist zur Diagnosestellung nicht erforderlich. Bei den verschiedenen klinischen Formen des Lupus erythematodes finden sich etwa in zwischen $25 \%$ und $75 \%$ der Fälle positive Testreaktionen, meist auf UV-B allein, oder zusätzlich auch auf UV-A (Lehmann P et al. J Am Acad Dermatol 1990; 22: 181 -187). Selbstverständlich kann eine Biopsie evtl. provozierter isomorpher Herde, ggf. mit direktem Immunfluoreszenz-Test, zur Diagnosesicherung erfolgen.

Bei Verdacht auf das Vorliegen einer phototoxischen oder photoallergischen Reaktion sollte ein belichteter Epikutantest (Photopatchtest) mit hälftiger UV-A-Belichtung nach 24 Stunden und Ablesung bis zu 72 Stunden danach erfolgen. Bei klinischem Verdacht sollte auch eine Porphyriediagnostik durchgeführt werden.

Rechtzeitig vor der Lichtsaison im nächsten Jahr können so die Weichen für eine spezifisch diagnosebezogene prophylaktische Therapie bzw. Prävention mittels „maßgeschneiderten“ Lichtschutzmitteln, UV-Gewöhnungstherapien oder Anwendung von Antimalariamitteln oder Immunsuppressiva gestellt werden.

\section{Lutz Kowalzick}

Klinik für Hautkrankheiten und Allergologie

HELIOS Vogtland-Klinikum

Plauen $\mathrm{GmbH}$

Postfach 100153

08505 Plauen

lutz.kowalzick@helios-kliniken.de 